\title{
Molecular and Immunogenic Properties of Apyrase SP01B and D7-Related SP04 Recombinant Salivary Proteins of Phlebotomus perniciosus from Madrid, Spain
}

\author{
Inés Martín-Martín, Ricardo Molina, and Maribel Jiménez \\ Unidad de Entomología Médica, Servicio de Parasitología, Centro Nacional de Microbiología, Instituto de Salud Carlos III, \\ Carretera Majadahonda-Pozuelo s/n, Majadahonda, 28220 Madrid, Spain
}

Correspondence should be addressed to Maribel Jiménez; mjimenez@isciii.es

Received 12 April 2013; Revised 26 July 2013; Accepted 16 August 2013

Academic Editor: Hirotaka Sakamoto

\begin{abstract}
Copyright ( 2013 Inés Martín-Martín et al. This is an open access article distributed under the Creative Commons Attribution License, which permits unrestricted use, distribution, and reproduction in any medium, provided the original work is properly cited.

Sand fly salivary proteins are on the spotlight to become vaccine candidates against leishmaniasis and to markers of exposure to sand fly bites due to the host immune responses they elicit. Working with the whole salivary homogenate entails serious drawbacks such as the need for maintaining sand fly colonies and the laborious task of glands dissection. In order to overcome these difficulties, producing recombinant proteins of different vectors has become a major task. In this study, a cDNA library was constructed with the salivary glands of Phlebotomus perniciosus from Madrid, Spain, the most widespread vector of Leishmania infantum in the Mediterranean basin. Analysis of the cDNA sequences showed several polymorphisms among the previously described salivary transcripts. The apyrase SP01B and the D7-related protein SP04 were successfully cloned, expressed in Escherichia coli, and purified. Besides, recombinant proteins were recognized by sera of hamsters and mice previously immunized with saliva through the exposure to uninfected sand fly bites. These results suggest that these two recombinant proteins conserved their immunogenic properties after expression in a prokaryote system. Therefore, this work contributes to expand the knowledge of P. perniciosus saliva that would be eventually used for the development of tools for vector control programs.
\end{abstract}

\section{Introduction}

Leishmaniasis is still one of the most important vectorborne diseases in terms of incidence as two million people per year are affected worldwide [1]. The causative agent of the aforementioned disease is Leishmania spp., a flagellate parasite which is transmitted by infected phlebotomine sand flies (Diptera: Psychodidae) during blood feeding. Arthropod saliva is actively involved in the transmission of pathogens to its host as it contains a complex cocktail of antihaemostatic and immunomodulatory molecules that are inoculated into the host skin during blood feeding of both infected and noninfected sand flies [2]. Concretely, sand fly salivary components are known to play an important role in the establishment of Leishmania spp. infection [3]. In the last years, research on sand fly salivary proteins has greatly increased, suggesting that salivary proteins could be successfully assayed both as anti-Leishmania vaccine candidates and as markers of exposure to sand flies $[4,5]$. As hosts are bitten, they develop both humoral and cellular responses against sand fly saliva [6]. Moreover, a positive correlation has been observed between the number of bites and antibody levels $[4,7,8]$. Therefore, host exposure to sand flies can be measured by evaluating humoral responses against salivary antigens. This methodology is being applied by mainly using salivary gland extracts [4, 9-12]. Recombinant salivary proteins have already been produced for sand fly species such as Lutzomyia longipalpis and Phlebotomus papatasi $[4,8,10,11,13,14]$. Some of these proteins have been already proved as good markers of exposure $[11,14]$. However, since salivary proteins display high specificity, it is necessary to produce immunogenic salivary proteins for other sand fly species.

In the western Mediterranean basin, Leishmania infantum is mainly transmitted by Phlebotomus perniciosus in an anthropozoonotic cycle where dogs have been traditionally considered the main reservoir [1]. However, other 
potential reservoirs such as hares (Lepus granatensis) have been recently involved in $L$. infantum transmission at a human leishmaniasis outbreak in Madrid [15, 16]. As a part of a management plan to control the disease in this environment, measuring exposure of reservoirs to the main vector involved, $P$. perniciosus, through the detection of anti-saliva antibodies, would be useful to evaluate whether actions taken to reduce leishmaniasis have been effective as previously done in India and Nepal [9].

In previous studies, our group described immunogenic salivary proteins of $P$. perniciosus and $P$ argentipes. These proteins were identified through the combination of twodimensional electrophoresis and Western blot with sera of mice and hamsters experimentally exposed to uninfected sand flies [17, 18]. Therefore, in this study, our aim was to obtain some of the $P$. perniciosus salivary immunogenic molecules as recombinant proteins, through a cDNA library from salivary glands, from $P$. perniciosus and subsequent studies of the immunogenicity of the purified salivary proteins. Moreover, several polymorphisms between transcripts of the P. perniciosus cDNA from Madrid were compared to the previously annotated ones that belonged to specimens from Italy [19].

\section{Material and Methods}

2.1. Sand Flies and Salivary Glands Collection. P. perniciosus sand flies were maintained at $27^{\circ} \mathrm{C}$ and $17: 7$ light-darkness photoperiod at the Medical Entomology Unit of the Instituto de Salud Carlos III (ISCIII), Madrid, Spain. This colony was established in 1987 from sand flies captured at a leishmaniasis endemic area of Madrid [20]. Salivary glands from recently emerged up to 1-day-old sand flies were dissected and stored in RNAlater (Invitrogen, San Diego, CA).

\subsection{Salivary Gland cDNA Library Construction. A cDNA} library was constructed with mRNA isolated from 165 salivary glands using the Micro-FastTrack mRNA isolation kit (Invitrogen, San Diego, CA). After isolation, mRNA was reverse transcrbted to $\mathrm{cDNA}$ and subsequently amplified by PCR following the instructions of the SMART CDNA library construction kit (Clontech). cDNAs were then fractionated by column chromatography before cloning. Directional cloning into $\lambda$ TriplEx2 vector (Clontech) was achieved through SfiIAB flanking sites incorporated by PCR during the cDNA amplification. The pool of cDNAs cloned into $\lambda$ TriplEx2 vector was packaged into phage particles following the manufacturer's instructions (Gigapack III Gold Packaging Extract, Agilent). The resulting cDNA library was amplified following general molecular biology protocols [21].

2.3. cDNA Library Screening, Sequencing, and Bioinformatics. After plating the library by infecting log phase XL1-blue cells (Clontech) in the presence of $\beta$-D-thiogalactoside (IPTG) and 5-bromo-4-chloro-indolyl- $\beta$-D-galactopyranoside (XGal), white plaques were randomly picked from the agar plates and analyzed by PCR using $\lambda$ TriplEx 2 primers (forward: $5^{\prime}$-TCCGAGATCTGGACGAGC-3' and reverse: $5^{\prime}$-TAATACGACTCACTATAGGGC-3' ${ }^{\prime}$ ). Recombinant phages which presented the greatest insert sizes were converted into pTriplEx2 plasmid through Cre recombinasemediated site-specific recombination. Plasmid DNA was then isolated and sequenced using the primer $5^{\prime} \lambda$ TriplEx LD: $5^{\prime}$-CTCGGGAAGCGCGCCATTGTGTTGGT- $3^{\prime}$ and the BigDye Terminator v3.1 Cycle Sequencing kit (PE Biosystems, Foster City, CA) in an ABI PRISM 3730XL DNA Analyzer (Applied Biosystems). Both strains of clones of interest were subsequently sequenced with reverse primer $3^{\prime} \lambda$ TriplEx LD: $5^{\prime}$-ATACGACTCACTATAGGGCGAATTGGCC- $3^{\prime}$. DNA electropherograms were manually inspected and corrected using Chromas program (McCarthy, Queensland, Australia). Sequence identities were determined by BLAST (http://blast.ncbi.nlm.nih.gov/). DNASTAR software (Lasergene, Madison, WI) was used for nucleotidic alignments (SeqMan program), and protein features were assigned by Protean tools. Amino acid sequences were aligned with ClustalW (http://www.ch.embnet.org/software/ClustalW.html) and refined using Boxshade server (http://www.ch.embnet .org/software/BOX_form.html). Glycosylation sites were predicted using NetNGlyc 1.0 Server (http://www.cbs.dtu.dk/ services/NetNGlyc/) and NetOGlyc 3.1 Server (http://www .cbs.dtu.dk/services/NetOGlyc/) for $\mathrm{N}$ - and O-glycosylation sites, respectively $[22,23]$. Phosphorylation sites prediction was done using NetPhos 2.0 Server (http://www.cbs.dtu .dk/services/NetPhos/) [24].

2.4. Cloning of Salivary Gland cDNAs. Salivary coding sequences of the apyrase SP01B and the D7-related protein SP04 were amplified by PCR from the corresponding pTriplEx2 plasmids using specific primers. cDNAs were cloned into PCR4-TOPO plasmid and transformed into TOP10 competent cells (Invitrogen, San Diego, CA). Restriction sites were incorporated by PCR from the recombinant PCR4TOPO vector. SalI (forward and reverse) was used as a restriction site (represented as bold letters): SP01B: $5^{\prime}$-GTCGACATGATATTGTTGAAATTG- $3^{\prime}$ and $5^{\prime}$-GTCGACTTACTTAATTCCTTTGGG-3' and SP04: $5^{\prime}$-GAGAGAGTCGACATGAATACCTTATTG- $3^{\prime}$ and $5^{\prime}$-GAGAGAGTCGACCTAATAATTTGTTAATG-3'. The primers were manually designed according to the sequences obtained. All primers were synthesized at the Genomic Unit of ISCIII. The highfidelity polymerase Pfu Turbo Hotstart (Stratagene, La Jolla, CA) was used in order to avoid point mutations. Coding sequences were excised from the plasmid by enzymatic digestion and ligated into pqE31 vector (Qiagen, Hilden, Germany). The recombinant expression vectors pqE31 were transformed into competent M15 (Qiagen, Hilden, Germany). All construction steps were verified by sequencing.

2.5. Expression and Purification of Recombinant Salivary Proteins. Different expression and purification conditions were tested in order to optimize the processes for these specific proteins. Cultures of M15 cells that contained the pqE31 recombinant plasmid for SP01B and SP04 were grown in Luria-Bertani medium containing ampicillin $(100 \mu \mathrm{g} / \mathrm{mL})$. 
Protein expression was induced by the addition of IPTG to a final concentration of $1 \mathrm{mM}$, and cultures were grown at $37^{\circ} \mathrm{C}$ for 3 hours. Bacterial cells were collected by centrifugation and lysed in $6 \mathrm{M}$ guanidine hydrochloride, $100 \mathrm{mM}$ $\mathrm{NaH}_{2} \mathrm{PO}_{4}, 10 \mathrm{mM}$ Tris- $\mathrm{HCl}$, and $\mathrm{pH}$ 8. Cell debris was then separated by centrifugation, and the resultant supernatant was submitted to affinity chromatography by using Ni-NTA Superflow resin in prepacked $5 \mathrm{~mL}$ columns. Proteins were purified under denaturing conditions following the manufacturer's instructions (Qiagen, Hilden, Germany). Protein refolding was done by removing urea through dialysis against PBS (SnakeSkin Dialysis Tubing $10 \mathrm{kDa}$ MWCO, Thermo Scientific, Goettingen, Germany). Proteins were then concentrated using the stirred ultrafiltration cell with $10 \mathrm{kDa} M W C O$ membranes (Millipore, Bedford, MA) and quantified by gel in comparison with BSA standards.

2.6. Western Blotting. Western blots were performed following standard protocols. Briefly, $1 \mu \mathrm{g}$ of recombinant protein was separated by SDS-PAGE and electroblotted onto a PVDF membrane which was incubated overnight in blocking buffer (3\% BSA, Sigma, St. Louis, CA; 2\% ECL Blocking reagent, Amersham, Piscataway, NJ). After washing, membranes were incubated for 2 hours with pooled sera $(1: 25)$ of either hamsters that were immunized against $P$. perniciosus saliva by the bite of uninfected sand flies through the exposure to 100 sand flies on a weekly schedule over 10 weeks [17] or mice exposed 13 times to 150 P. perniciosus (unpublished). Goat anti-hamster IgG and goat anti-mouse IgG peroxidaseconjugated antibodies (1:3500, Southern Biotech, Birmingham, AL and 1:500, AbD Serotec, resp.) were used, and immunogenic proteins were visualized by $\mathrm{CN} / \mathrm{DAB}$ reagent following the manufacturer's instructions (Thermo Scientific, Goettingen, Germany). Experiments with sera of immunized and nonimmunized animals were carried out in parallel. For His-tag detection, mouse anti-RGS-His antibodies (1:3000, Qiagen, Hilden, Germany) were used in combination with the goat anti-mouse IgG peroxidase-conjugated antibodies (AbD Serotec).

\section{Results and Discussion}

3.1. cDNA Library Analysis. The expression library showed a titer of $3.6 \times 10^{6}$ and $17.6 \%$ of nonrecombinant clones. The amplified library displayed a larger number of independent clones $\left(1.2 \times 10^{12}\right)$ and a low number of nonrecombinant clones (1.92\%). Therefore, this amplified library was used for subsequent analysis and cloning processes. 201 white plaques were randomly picked from IPTG/X-Gal plates and analyzed by PCR. 36 out of 201 plaques showing the greatest insert size were selected, converted into pTriplEx2, and further sequenced. Among the 25 complete transcripts, they were identified mostly as apyrases (SP01, SP01B), D7-related proteins (SP04, SP04B), yellow proteins (SP03B), ParSP25-like proteins (SP08), lufaxin-like proteins (SP06), and PpSP15-like proteins (SP02, SP09, and SP11). Complete sequences were annotated at EMBL nucleotide database, and NCBI accession numbers are shown in Table 1.
Overall, high degree of conservancy was found among the salivary transcripts we sequenced, and their best matches available in nonredundant databases were obtained from a cDNA library from P. perniciosus from Italy [19]. This finding is in contrast to the high level of divergence found for the salivary protein maxadilan of $L$. longipalpis from distinct locations [25].

In the case of maxadilan, the high level of polymorphisms elicits variant-specific antibodies with little cross-reactivity, and it has been suggested that sand flies may have evolved diversity in maxadilan as a strategy to evade the host immune response against this essential vasodilator peptide that facilitates blood feeding of $L$. longipalpis. Balancing selection might be maintaining many maxadilan alleles with equivalent vasodilatory potencies $[25,26]$. On the other hand, our results match the description of a high degree of conservancy among salivary transcripts in two geographically distant Phlebotomus duboscqi sand fly populations in Africa, and it was suggested that sand flies belonging to the genus Phlebotomus show greater degree of conservancy than Lutzomyia spp. [27]. Following this trend, in previous experiments of our group, we did not find qualitative differences in the salivary protein profile among three colonies of $P$. perniciosus collected from different areas of Spain and reared under identical laboratory conditions [17]. Moreover, a recent comparison between transcripts from cDNA libraries constructed with $P$. papatasi strains from Israel and Tunisia showed a high level of conservancy [28]. Indeed, the high degree of conservancy found between the salivary transcripts of $P$. perniciosus from Spain and Italy may be a reflection of little evolutionary pressure from the host immune response on the analyzed salivary proteins in contrast with previous observations of maxadilan. Moreover, studies on the evolution of apyrase from several Phlebotomus species show high degree of conservancy, and the geographical pattern of genetic variation was consistent with neutral demographic processes mainly regional isolation and isolation by distance resulting from the changes that occurred during the late Pleistocene [29]. In the case of secretary salivary proteins, it is possible that the presence of multiple copies reflects an adaptation to increase the production of these important proteins for sand flies [30]. Salivary peptides often occur within and between gene families, for which there is evidence for overlapping functions [19]. Redundancy is a common property among salivary proteins of sand flies and other arthropods [17, 31], and it has been suggested that it might play a role in ensuring blood-feeding success [32].

Therefore, several polymorphisms were found when comparing the sequenced salivary transcripts in this work with their corresponding annotated transcripts. Although these polymorphisms are expected among populations, we aimed to in silico study in detail whether these polymorphisms could lead to changes in phosphorylation and glycosylation processes, structure, immunogenic properties, and functionality through bioinformatics-predictive programs. In order to confirm these single nucleotide polymorphisms (SNPs) both $5^{\prime}$ and $3^{\prime}$ strands were sequenced and aligned. Most of the SNPs were located among the $5^{\prime}$ and $3^{\prime}$ untranslated regions (UTRs) which are known for the high variability [33]. 


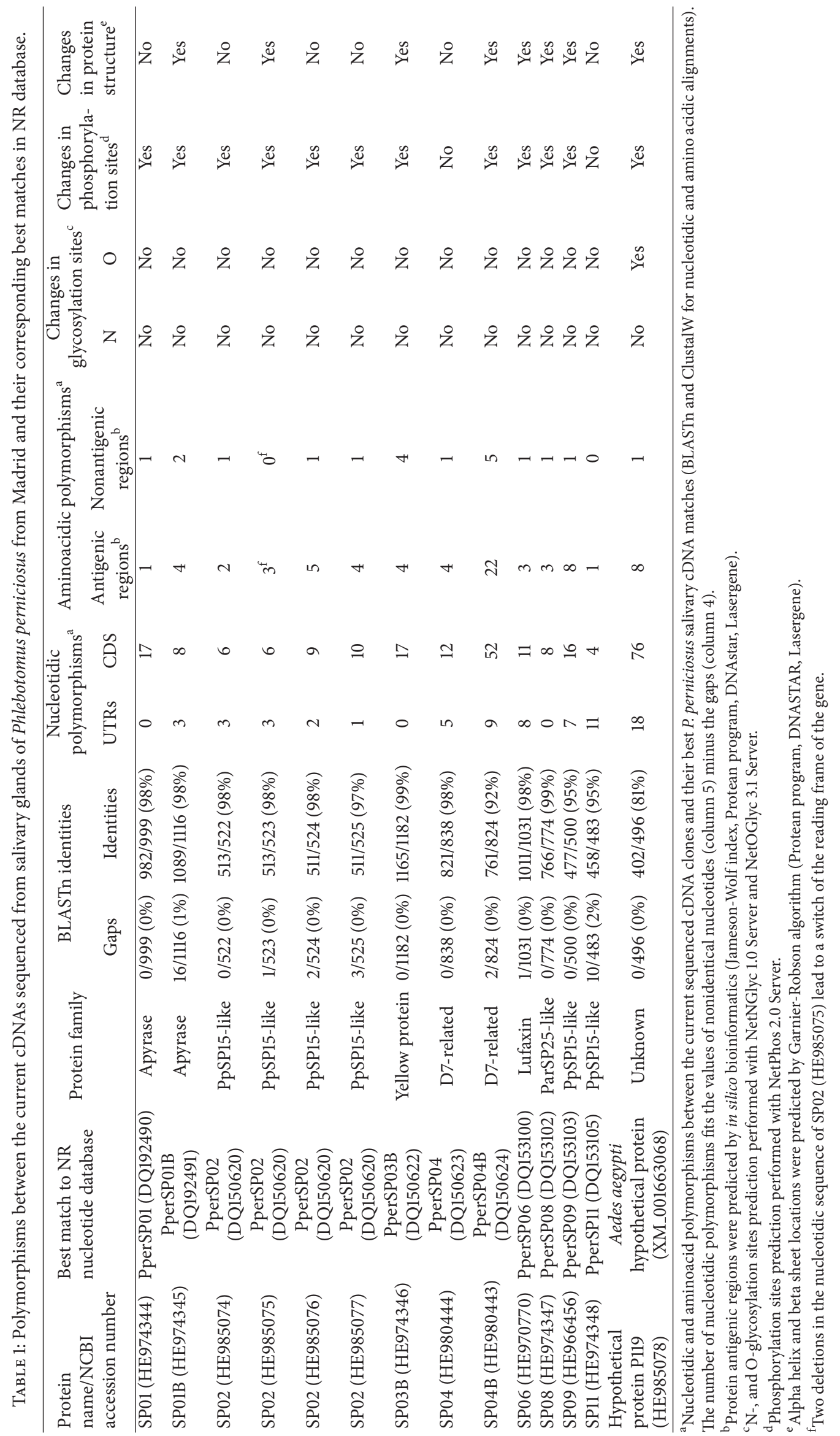


On the other hand, several SNPs were found on the translated region, and some of them resulted in amino acid changes. Substitutions in coding regions may influence protein structure, and therefore electrostatic forces and ligand affinity may involve changes in protein function [34]. Prediction of locations of alpha and beta regions, as well as the antigenic index and phosphorylation sites for the sequenced proteins, confirmed the presence of several changes from their best matches in databases as shown in Table 1. Polymorphisms found in SP01B and SP04 resulted in changes of the antigenic index from positive to negative values and vice versa when compared to the Italian strain (located at $\mathrm{T}^{166}$ for SP01B and $\mathrm{R}^{51}$ for SP04). In the case of phosphorylation residues, most of the analyzed transcripts presented changes regarding to the $P$. perniciosus Italian strain. For instance, in the apyrase sequence SP01B, the amino acids $\mathrm{T}^{163}$ and $\mathrm{Y}^{148}$ were predicted to be phosphorylated in the Italian strain but not in the Spanish one (Figure 1). Although point mutations can change the ability of the protein to bind carbohydrates, the SNPs were not located at the $\mathrm{N}$ - and $\mathrm{O}$-glycosylation sites as in silico predicted through bioinformatic programs (Table 1).

A description of the salivary proteins from $P$. perniciosus Spanish strain is listed below.

3.1.1. Apyrases. SP01 and SP01B belong to the protein family of Cimex apyrases which are typically found in sand flies and bed bugs. These enzymes hydrolyze nucleotide di- and triphosphates to orthophosphates and mononucleotides and act as potent antihaemostatic factors [13]. Transcripts coding for SP01 and SP01B in our library are conserved with the respective genes from $P$. perniciosus from Italy (Figure 1).

3.1.2. Odorant-Binding-Related Proteins. In sand flies two salivary protein families are included among the odorantbinding protein superfamily; the D7-related proteins and the PpSP15-like proteins $[35,36]$.

The SP04 and SP04B are classified as D7-related proteins. D7-related proteins are widely distributed in the saliva of haematophagous Diptera. One of the first cloned proteins from the saliva of Aedes aegypti was arbitrarily called D7 [37], and since then similar proteins have been identified in the saliva of other Nematocera such as mosquitoes, black flies, Culicoides, and sand flies [36]. Further studies showed that the odorant-binding-protein superfamily had a multigene organization and due to gene duplication processes produced two distinct forms of proteins called long and short forms [38]. Sand fly D7-related proteins are related to the long form [39].

In mosquitoes, D7 proteins are able to bind biogenic amines and leukotrienes, in addition to various components of the coagulation cascade, thus interfering with the haemostatic and host immune responses [40]. The role they play in sand flies has not yet been clarified however, their great representation at both transcriptomic and proteomic levels $[17-19,28,41]$ and the conservation of the cysteinyl leukotrienes-binding motifs [42] suggest an involvement in counteracting the coagulation cascade [41]. Concretely, the binding site for thromboxane A2 (TXA2) analogs in A. stephensi D7 protein (GenBank ID: 315583502) lies in a hydrophobic pocket in the N-terminal domain which accommodates a large portion of the fatty acid chain. Tyrosine at position 52 in A. stephensi D7 protein is known to stabilize the TXA2 analogs through hydrogen bonds [42]. In $A$. aegypti D7 protein (GenBank ID: PDB:3DXL_A), a mutation of this position from tyrosine to phenylalanine leads to a failure to bind TXA2 analogs [43]. In sand flies, most of the D7-related proteins conserved a residue of tyrosine in this position $\left(\mathrm{Y}^{65}\right)$; however, both SP04 from P. perniciosus Spanish and Italian strains contain a phenylalanine in that position (Figure 2). Moreover, in the D7-related protein SP04 from the P. perniciosus Spanish strain (GenBank ID: CCK73754), one of the cysteinyl leukotriene-binding motifs located at $\mathrm{R}^{51}$ showed a polymorphism (Figure 2 ). However, without experimental confirmation of the functionality of D7-related proteins in sand flies, these changes in the binding motif remain to be elucidated. The D7-related protein SP04B from the Spanish strain (GenBank ID: HE980443) showed two deletions between $\mathrm{T}^{546}$ and $\mathrm{A}^{547}$ that lead to a switch in the reading frame of the gene and an earlier stop codon $\left({ }^{546} \mathrm{TAG}^{548}\right.$, data not shown). The predicted protein should appear in the polyacrylamide gels at a theoretical molecular weight (Mw) of $20.7 \mathrm{kDa}$, however, it had not been found at that Mw $[17,19]$. In order to check whether all SP04B clones present in this library included this mutation, we amplified the SP04B gene with primers flanking the CDS, the cDNA library as a template, and further sequenced the extracted DNA. We found the complete sequence for SP04B without that mutation, confirming that in our library both clones are present.

SP02, SP09, and SP11 belong to the PpSP15-like family which have been found only in sand flies and represent the most abundant family among sand fly salivary proteins [19, 41, 44]. PpSP15-like proteins were described as highly polymorphic and derived from a multicopy gene family [19, 30]. The sequences of these proteins show a high degree of variability in addition to certain conserved cysteine residues [28]. In P. papatasi, large numbers of variants of the PpSP15 gene were observed, and differences between alleles were small and resulted in only few amino acid substitutions [30]. Therefore, polymorphisms within transcripts are expected, as shown in other species. Some transcripts, such as SP02, were found to be highly representative within the cDNA library (Figure 3). One of the sequenced clones of SP02 (GenBank ID: HE985075) presented a single nucleotide deletion between $\mathrm{A}^{340}$ and $\mathrm{T}^{341}$ that leads to a switch in the reading frame of the gene. As a consequence, the putative-translated protein (GenBank ID: CCM43815) would differ in sequence and length since it contains an earlier stop codon $\left({ }^{417} \mathrm{TGA}^{419}\right.$, Figure 3). Although these changes do not seem to affect its immunogenicity, as the polymorphisms were found within an area of low antigenicity according to the Jameson-Wolf index (data not shown), the function of this protein family remains unknown. Interestingly, immunization with P. papatasi SP15 protected mice from cutaneous leishmaniasis caused by Leishmania major [45]. 


Dub_ABI20151
Pap_AF261768
Ser_ADJ54110
Ara_ACS93496
Ari_AAX56357
PerIta_ABB00907
PerSpa_CCK33660
Tob_ADJ54077
Arg_ABA12135
Lon_AF131933
Int_AFP99246
Aya_BAM69107
Hum_Q8WVQ1
Dro_CAL26008
Cim_AAD09177

Dub_ABI20151 Pap_AF261768 Ser_ADJ54110 Ara_ACS93496 Ari_AAX56357 PerIta_ABB00907 PerSpa_CCK33660 Tob_ADJ54077 Arg_ABA12135 Lon_AF131933 Int_AFP99246 Aya_BAM69107 Hum_Q8WVQ1 Dro_CAL26008 Cim_AAD09177

Dub_ABI20151 Pap_AF261768 Ser_ADJ54110 Ara_ACS93496 Ari_AAX56357 PerIta_ABB00907 PerSpa_CCK33660 Tob_ADJ54077 Arg_ABA12135 Lon_AF131933 Int_AFP99246 Aya_BAM69107 Hum_Q8WVQ1 Dro_CAL26008 Cim_AAD09177

22
22
22
21
21
22
22
22
22
22
18
22
69
77
30
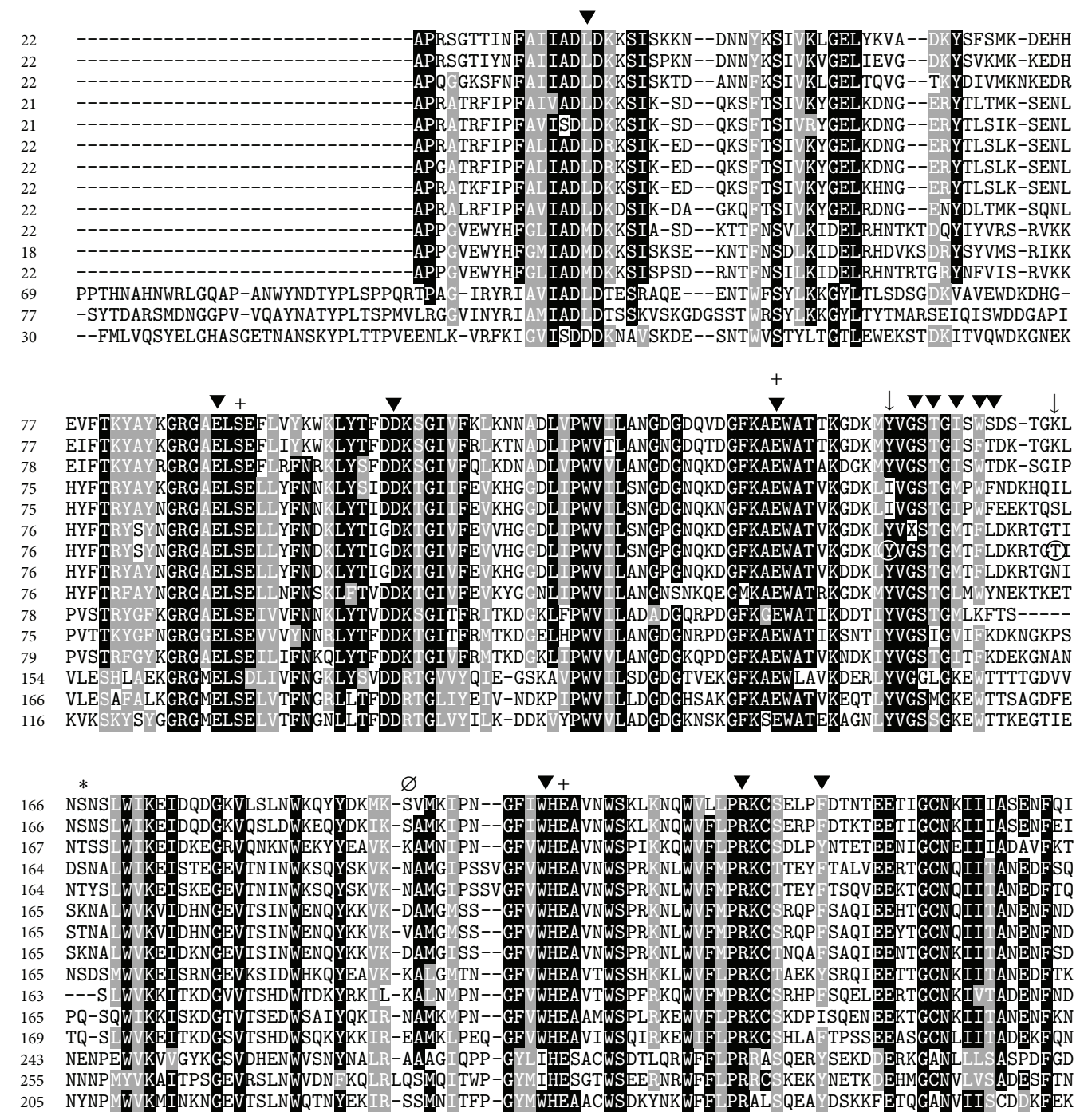

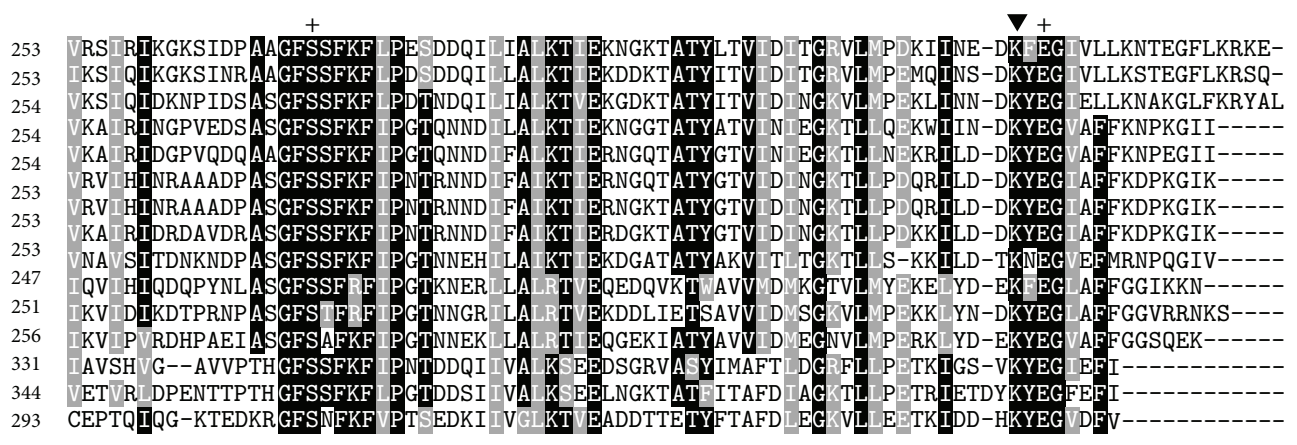

Figure 1: Multiple sequence alignment of apyrases from sand flies and other related sequences: L. longipalpis (Lon), Lutzomyia intermedia (Int), Lutzomyia ayacuchensis (Aya), Phlebotomus arabicus (Ara), Phlebotomus ariasi (Ari), P. perniciosus Italian strain (PerIta), P. perniciosus Spanish strain (PerSpa), Phlebotomus tobbi (Tob), Phlebotomus argentipes (Arg), P. duboscqi (Dub), P. papatasi (Pap), Phlebotomus sergenti (Ser), Homo sapiens (Hum), Drosophila melanogaster (Dro), and Cimex lectularius (Cim). Accession numbers are indicated in the sequence name. Sequences without a signal peptide were aligned with ClustalW and refined using Boxshade server, and the percentage of the identities or similarities that must agree for shading was set at $80 \%$. Black background shading represents identical amino acids, and grey shading designates similar amino acids while white shading indicates no similarity. $(*)$ and $(\varnothing)$ indicate changes in the prediction of the antigenic index and secondary structure, respectively, between P. perniciosus Spanish and Italian strains as performed by Protean (DNASTAR, Lasergene). $(\downarrow)$ signs above amino acids indicate changes in phosphorylation sites as predicted by NetPhos 2.0 Server, and the amino acid affected by the prediction on the phosphorylation site is encircled. Binding sites of nucleotides and $\mathrm{Ca}^{2+}$ are represented by $(\boldsymbol{\nabla})$ and $(+)$, respectively, as predicted for the human apyrase [52]. 


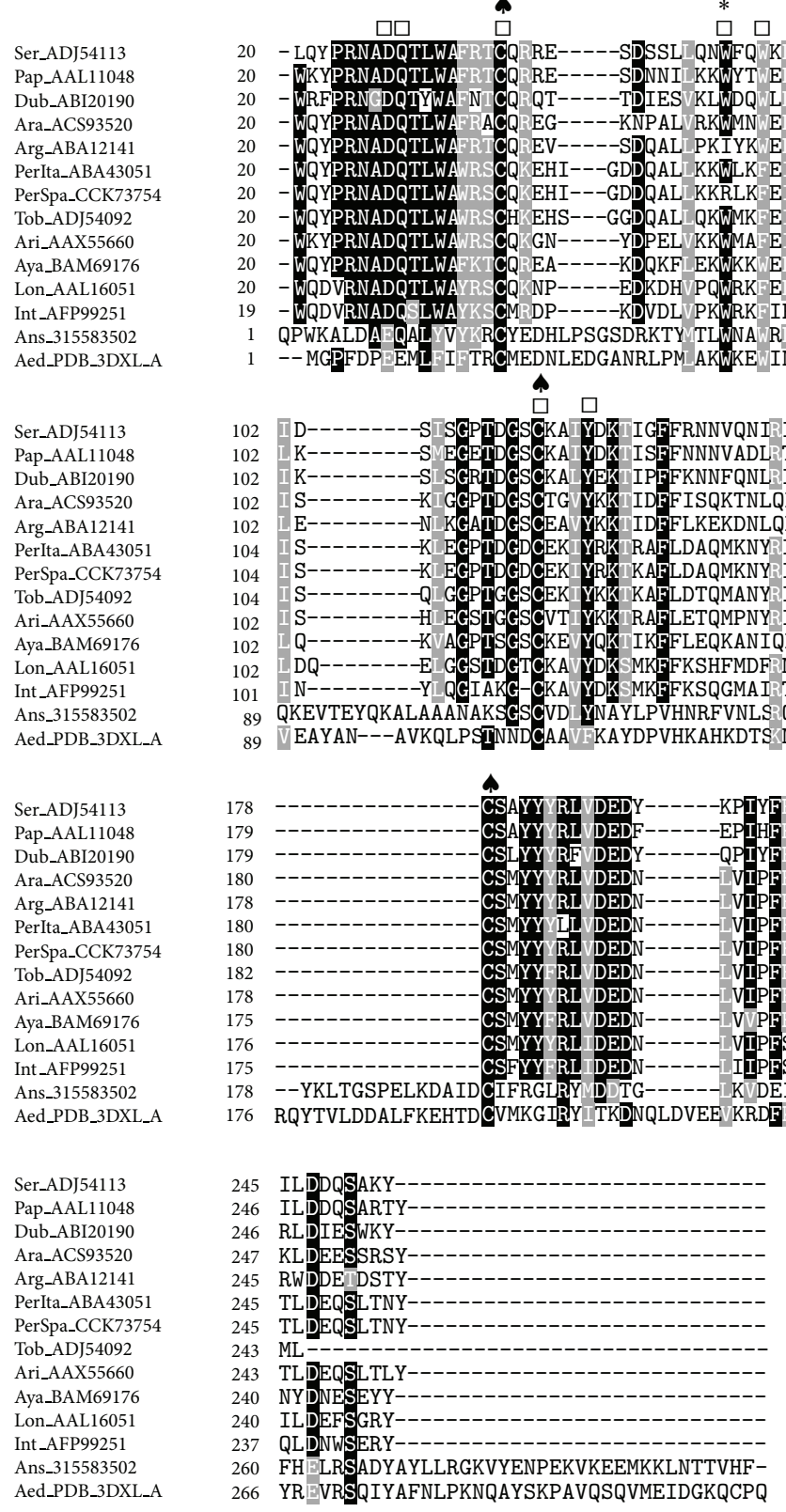

Figure 2: Multiple sequence alignment of D7-related proteins from sand flies and other related sequences: L. longipalpis (Lon), L. intermedia (Int), L. ayacuchensis (Aya), P. arabicus (Ara), P. ariasi (Ari), P. perniciosus Italian strain (PerIta), P. perniciosus Spanish strain (PerSpa), P. tobbi (Tob), P. argentipes (Arg), P. duboscqi (Dub), P. papatasi (Pap), P. sergenti (Ser), A. stephensi (Ans), and A. aegypti (Aed). Accession numbers are indicated in the sequence name. Sequences without a signal peptide were aligned with ClustalW and refined using Boxshade server, and the percentage of the identities or similarities that must agree for shading was set at $80 \%$. Black background shading represents identical amino acids, and grey shading designates similar amino acids, while white shading indicates no similarity. $(*)$ indicates changes in the prediction of the antigenic index between P. perniciosus Spanish and Italian strains as performed by Protean (DNASTAR, Lasergene). ( $\mathbf{\uparrow})$ denotes conserved cysteine residues. The cysteinyl leukotriene-binding motif is indicated by ( $\square$ ) [42], and the mutation of one of the amino acids that integrate this motif is designated by $(\boldsymbol{\nabla})$. Tyrosine in position 52 in A. stephensi stabilizes TXA2 analogs and is highlighted with (o).

3.1.3. Yellow Proteins. The yellow proteins are found in the saliva of insects, and they were named after observations in Drosophila, where mutation of a given gene gave a yellow phenotype, indicating that they are involved in melanization processes and pigmentation [46]. In sand flies, yellow proteins are widely represented at both transcriptomic and proteomic levels, showing molecular weights around $41-45 \mathrm{kDa}$ and a wide range of isoelectric points [17, 19, 35, 41, 44]. Recently, it has been demonstrated that yellow proteins from $L$. longipalpis are able to bind and therefore inhibit the effects of several biogenic amines such as serotonin, norepinephrine, epinephrine, and histamine. Therefore, 


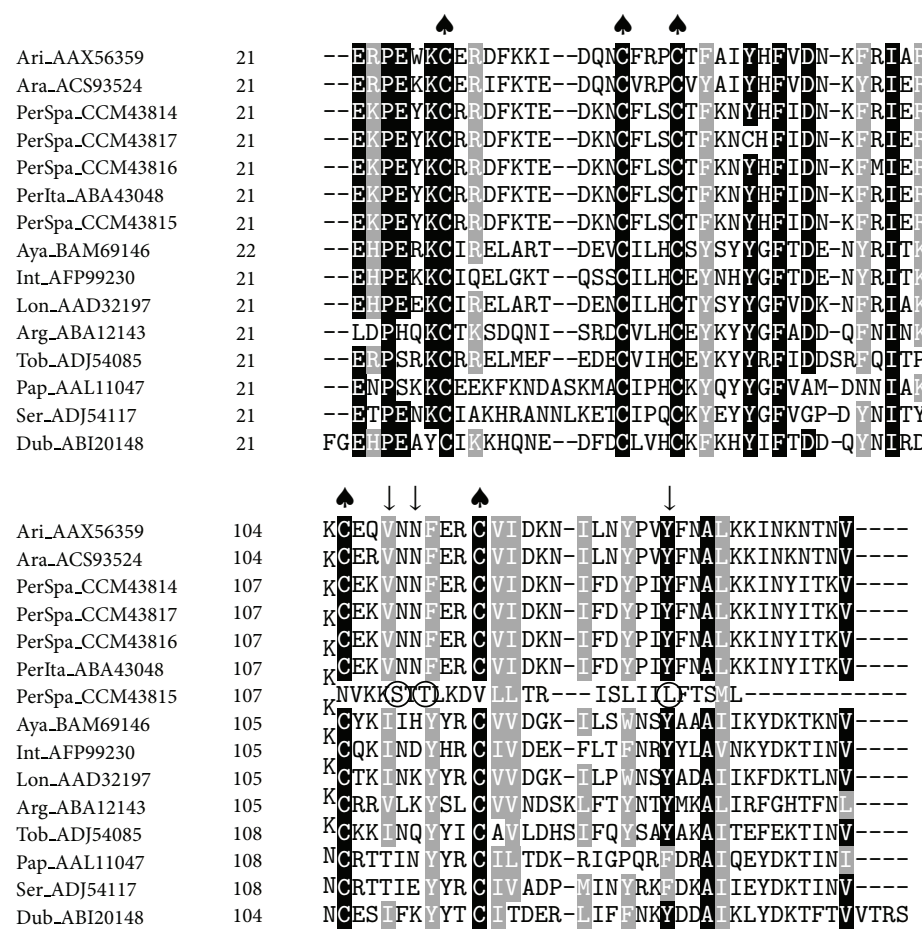

FIGURE 3: Multiple sequence alignment of PpSP15-like proteins from sand flies: L. longipalpis (Lon), L. intermedia (Int), L. ayacuchensis (Aya), P. arabicus (Ara), P. ariasi (Ari), P. perniciosus Italian strain (PerIta), P. perniciosus Spanish strain (PerSpa), P. tobbi (Tob), P. argentipes (Arg), P. duboscqi (Dub), P. papatasi (Pap), and P. sergenti (Ser). Accession numbers are indicated in the sequence name. Sequences without a signal peptide were aligned with ClustalW and refined using Boxshade server, and the percentage of the identities or similarities that must agree for shading was set at $80 \%$. Black background shading represents identical amino acids, and grey shading designates similar amino acids, while white shading indicates no similarity. ( $($ ) indicates changes in the prediction of the secondary structure between $P$. perniciosus Spanish and Italian strains as performed by Protean (DNASTAR, Lasergene). $(\downarrow)$ signs above amino acids indicate changes in phosphorylation sites as predicted by NetPhos 2.0 Server, and the amino acid affected by the prediction on the phosphorylation site is encircled. ( $\mathbf{A})$ denotes conserved cysteine residues.

they act as vasodilators and as inhibitors of platelet activation, itch, and pain [47]. Yellow proteins are conserved among sand fly species as shown in Figure 4.

3.1.4. Par25-Like Proteins. This protein family displays a molecular weight around $25 \mathrm{kDa}$ and an isoelectric point of 4.4-5 due to the great proportion of acidic residues (Figure 5). There are conserved regions rich in certain amino acid residues, and this protein family has been identified in Adlerius ( $P$. arabicus), where it represents the second most abundant protein family in the salivary glands cDNA, and Larroussius (P. ariasi, P. perniciosus, and P. tobbi) [19, 41, $44,48]$. Although its function is still unknown, several members of the sand fly ParSP25-like family stand to be highly immunogenic. Concretely, P. perniciosus SP08 is highly recognized by the sera of mice, hamsters, and dogs exposed to the bites of this vector $[7,17]$. Moreover, a plasmid encoding $P$. ariasi SP25 strongly elicits both humoral and delayed-type hypersensitivity responses in mice [48].

3.1.5. Lufaxin-Like Proteins. SP06 from P. perniciosus Spanish strain (GenBank ID: CCK18305), a component of the $33 \mathrm{kDa}$ family protein, shares $44 \%$ sequence identity with lufaxin from L. longipalpis (GenBank ID: AAS05319, Figure 6) whose antithrombotic and anti-inflammatory effects have been recently described [49]. These alkaline proteins of molecular weight around $32-36 \mathrm{kDa}$ seem to be specific for sand flies, and they have been identified in species of both genera Phlebotomus and Lutzomyia [19, 28, 35, 41, 49-51].

The transcript coding for a hypothetical protein P119 (GenBank ID: HE985078) was found among the sequenced cDNAs. This predicted protein shares homology with several hypothetical proteins of different insects, being the best match from A. aegypti (GenBank ID: XM_001663068). However, since it lacks signal peptide, it will not probably be secreted into the salivary gland lumen and would possibly be found in the cells of the gland walls.

\subsection{Cloning, Expression, and Purification of Salivary Proteins.} cDNA sequences of the salivary proteins SP01B and SP04 were successfully cloned in frame into expression vectors as confirmed by sequencing. Different expression conditions of the apyrase SP01B (37.3 kDa) and the D7-related protein SP04 $(28.9 \mathrm{kDa})$ were assayed, and the best results were obtained when we induced cultures with $1 \mathrm{mM}$ IPTG during 3 hours at $37^{\circ} \mathrm{C}$. Batch purification of both $6 \times$ His-tagged proteins was achieved by His-tag affinity chromatography using Super flow Ni-NTA resin under denaturing conditions. Adequate 
PerIta_ABA43050 PerSpa_CCK33661 Tob_ADJ54080 Ari_AAX44093 Ara_ACS93501 Arg_ABA12136 Aya_BAM69185 Int_AFP99235 Ser_ADJ54123 Pap_AGE83095 Dub_ABI20172 Lon_AAS05318 Ang_XP_312785_2 Dro_XP_002097461

PerIta_ABA43050 PerSpa_CCK33661 Tob_ADJ54080 Ari_AAX44093 Ara_ACS93501 Arg_ABA12136 Arg_ABA12136 Aya_BAM69185 Int_AFP99235 Ser_ADJ54123
Pap_AGE83095 Dub_ABI20172 Lon_AAS05318 Dro_XP_002097461 Ang_XP_312785_2

PerIta_ABA43050 PerSpa_CCK33661 Tob_ADJ54080 Ari_AAX44093 Ara_ACS93501 Arg_ABA12136 Aya_BAM69185 Int_AFP99235 Ser_ADJ54123 Pap_AGE83095 Pap_AGE83095 Dub_ABI20172 Lon_AAS05318 Dro_XP_002097461

PerIta_ABA43050 PerSpa_CCK33661 Tob_ADJ54080 Ari_AAX44093 Ara_ACS93501 Arg_ABA12136 Aya_BAM69185 Aya_BAM69185 Int_AFP99235 Ser_ADJ54123
Pap_AGE83095 Dub_ABI20172 Lon_AAS05318 Ang_XP_312785_2 Dro_XP_002097461
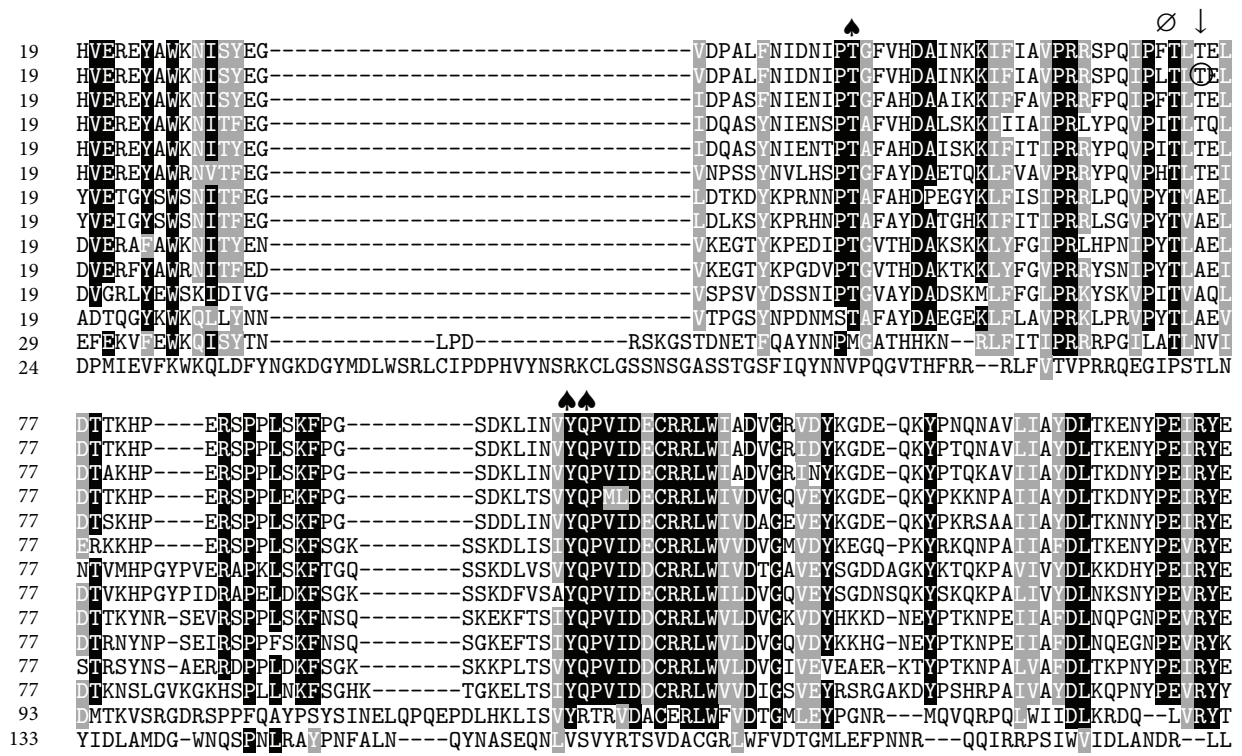

A

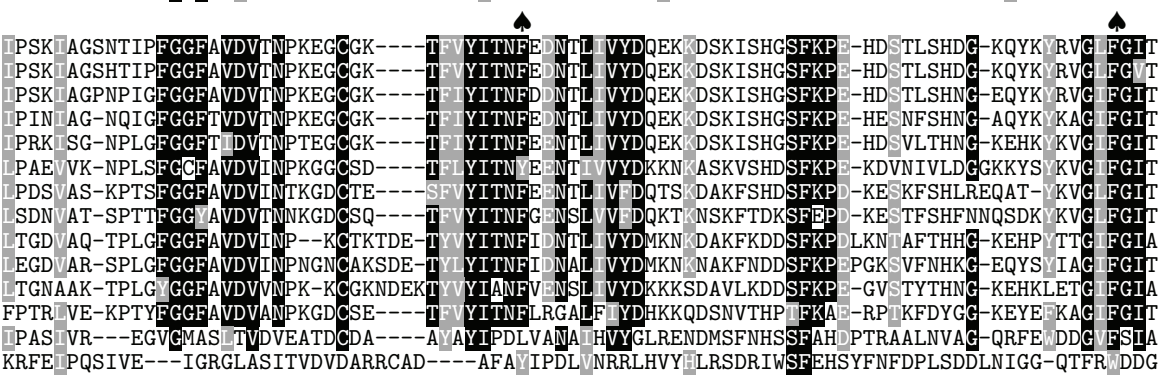
KRFE IPQSIVE---IGRGLASITVDVDARRCAD----AFAYIPDLVNRRLHVY

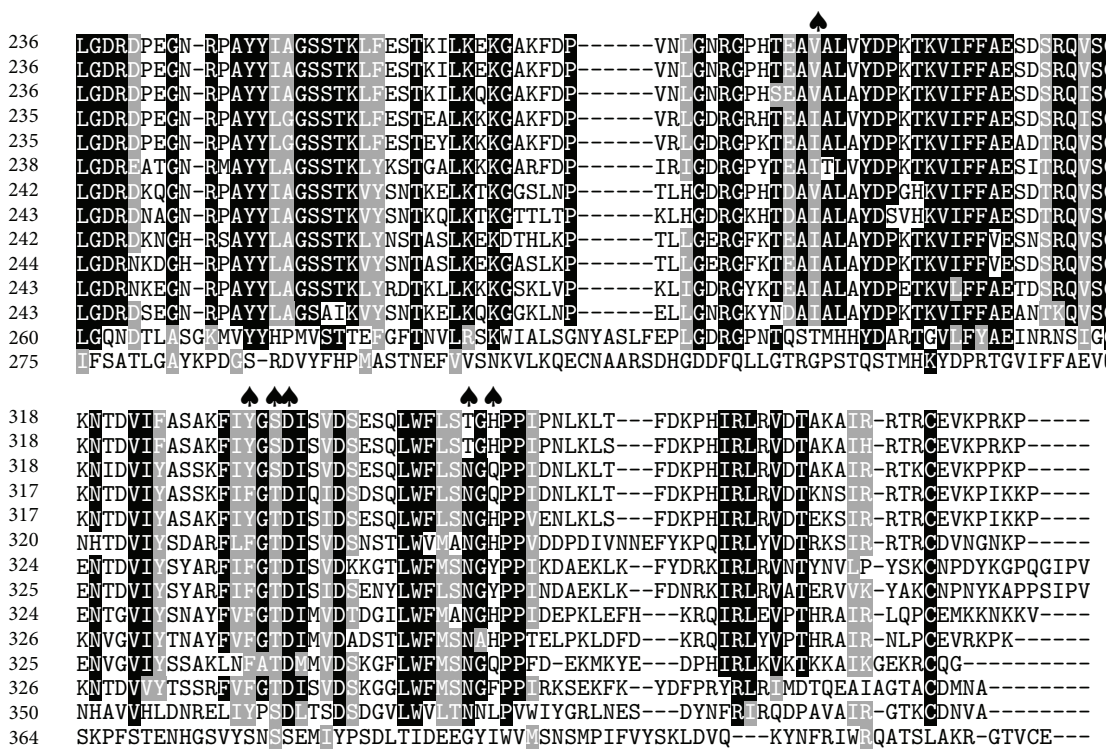

Figure 4: Multiple sequence alignment of yellow proteins from sand flies and other related sequences: L. longipalpis (Lon), L. intermedia (Int), L. ayacuchensis (Aya), P. arabicus (Ara), P. ariasi (Ari), P. perniciosus Italian strain (PerIta), P. perniciosus Spanish strain (PerSpa), P. tobbi (Tob), P. argentipes (Arg), P. duboscqi (Dub), P. papatasi (Pap), P. sergenti (Ser), Anopheles gambiae (Ang), and D. melanogaster (Dro). Accession numbers are indicated in the sequence name. Sequences without a signal peptide were aligned with ClustalW and refined using Boxshade server, and the percentage of the identities or similarities that must agree for shading was set at $80 \%$. Black background shading represents identical amino acids, and grey shading designates similar amino acids, while white shading indicates no similarity. $(\varnothing)$ indicates changes in the prediction of the secondary structure between P. perniciosus Spanish and Italian strains as performed by Protean (DNASTAR, Lasergene). $(\downarrow)$ signs above amino acids indicate changes in phosphorylation sites as predicted by NetPhos 2.0 Server, and the amino acid affected by the prediciton on the phosphorylation site is encircled. ( $\mathbf{A})$ denotes conserved amino acids contained in the ligand-binding pocket. 


Dub_ACS93514
Ara_ACS93513
Ari_AAX55664
PerIta_ABA43056
PerSpa_CCK33662
Tob_ADJ54100

Dub_ACS93514
Ara_ACS93513
Ari_AAX55664
PerIta_ABA43056
PerSpa_CCK33662
Tob_ADJ54100

Dub_ACS93514
Ara_ACS93513
Ari_AAX55664
PerIta_ABA43056
PerSpa_CCK33662
Tob_ADJ54100

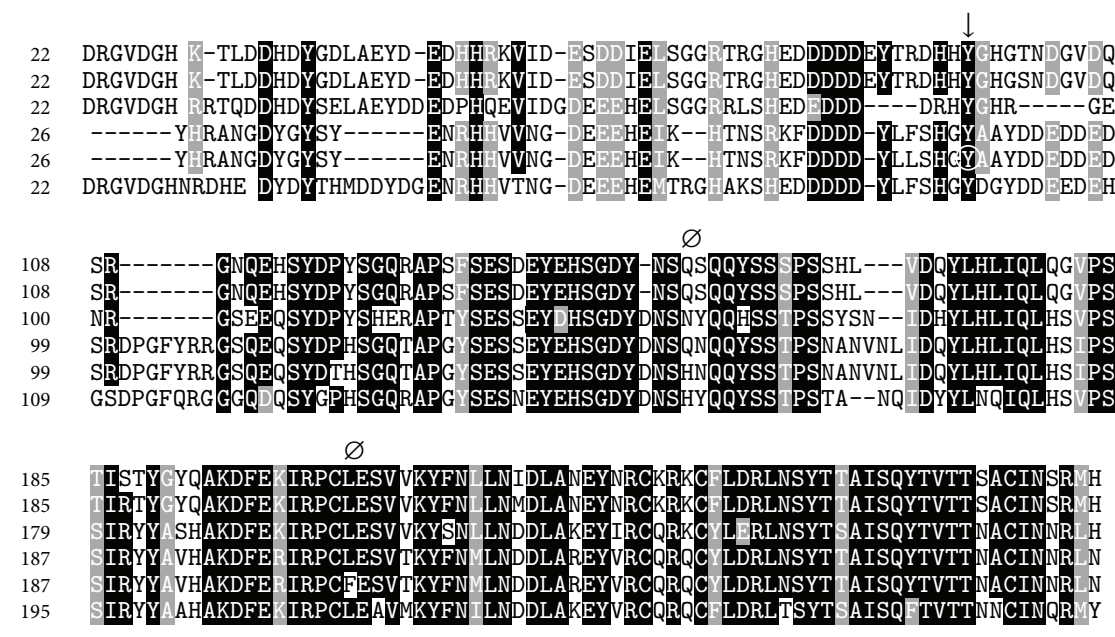

FIgure 5: Multiple sequence alignment of Par25-like proteins from sand flies: P. arabicus (Ara), P. ariasi (Ari), P. perniciosus Italian strain (PerIta), and P. perniciosus Spanish strain (PerSpa), P. tobbi (Tob), P. duboscqi (Dub). Accession numbers are indicated in the sequence name. Sequences without signal peptide were aligned with ClustalW and refined using Boxshade server, and the percentage of the identities or similarities that must agree for shading was set at $80 \%$. Black background shading represents identical amino acids, and grey shading designates similar amino acids, while white shading indicates no similarity. $(\varnothing)$ indicates changes in the prediction of the secondary structure between P. perniciosus Spanish and Italian strains as performed by Protean (DNASTAR, Lasergene). ( $\downarrow$ ) signs above amino acids indicate changes in phosphorylation sites as predicted by NetPhos 2.0 Server, and the amino acid affected by the prediciton on the phosphorylation site is encircled.

PerSpa_CCK18305
PerIta_ABA43054
Lon_AAS05319
PerSpa_CCK18305
PerIta_ABA43054
Lon_AAS05319
PerSpa_CCK18305
PerIta_ABA43054
Lon_AAS05319
PerSpa_CCK18305
PerIta_ABA43054
Lon_AAS05319

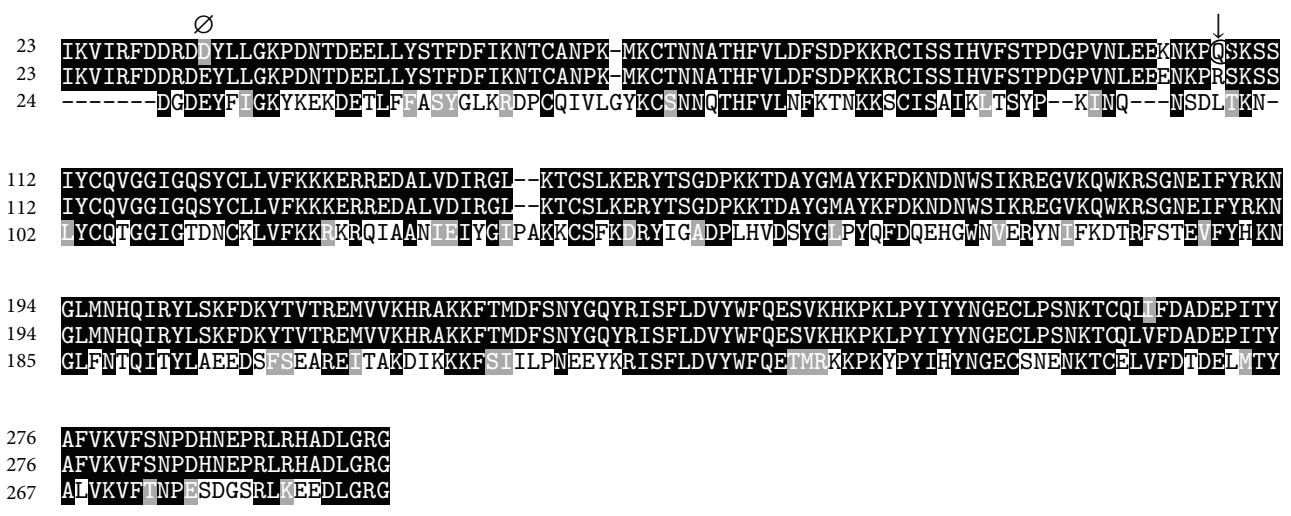

Figure 6: Alignment of SP06 from P. perniciosus Italian strain (PerIta), P. perniciosus Spanish strain (PerSpa), and lufaxin from L. longipalpis (Lon). Accession numbers are indicated in the sequence name. Sequences without a signal peptide were aligned with ClustalW and refined using Boxshade server, and the percentage of the identities or similarities that must agree for shading was set at $80 \%$. Black background shading represents identical amino acids, and grey shading designates similar amino acids, while white shading indicates no similarity. ( $\varnothing$ ) indicates changes in the prediction of the secondary structure between P. perniciosus Spanish and Italian strains as performed by Protean (DNASTAR, Lasergene). $(\downarrow)$ signs above amino acids indicate changes in phosphorylation sites as predicted by NetPhos 2.0 Server, and the amino acid affected by the prediciton on the phosphorylation site is encircled.

expression pattern was attained for these salivary proteins as the corresponding Coomassie blue-stained bands matched the theoretical Mw, and Western blot revealed the presence of His-tagged protein (Figure 7 and Figure 8(b), resp.).

The apyrase SP01B and the D7-related protein SP04 were chosen for expression and purification since they had previously described as antigens for mice, hamsters, and dogs $[7,17]$, and they are highly represented in the saliva as seen in P. perniciosus salivary proteome $[17,19]$. Apyrases have been pointed as good vaccine candidates since they display putative MHC epitopes, and a recombinant apyrase of $P$. ariasi produced protective cellular DTH responses in mice
[27, 29, 48]. Besides, apyrases are conserved proteins among sand flies and therefore good candidates for a wider vaccine [29].

3.3. Immunogenicity of Recombinant SP01B and SP04. Both recombinant proteins SP01B and SP04 were recognized by the sera of hamsters and mice exposed to sand fly bites in Western blot (Figure 8(a)).

Therefore, immunogenicity of the recombinant proteins was conserved for at least these animals after expression in a prokaryote system. Thus, we hypothesize that part of their 


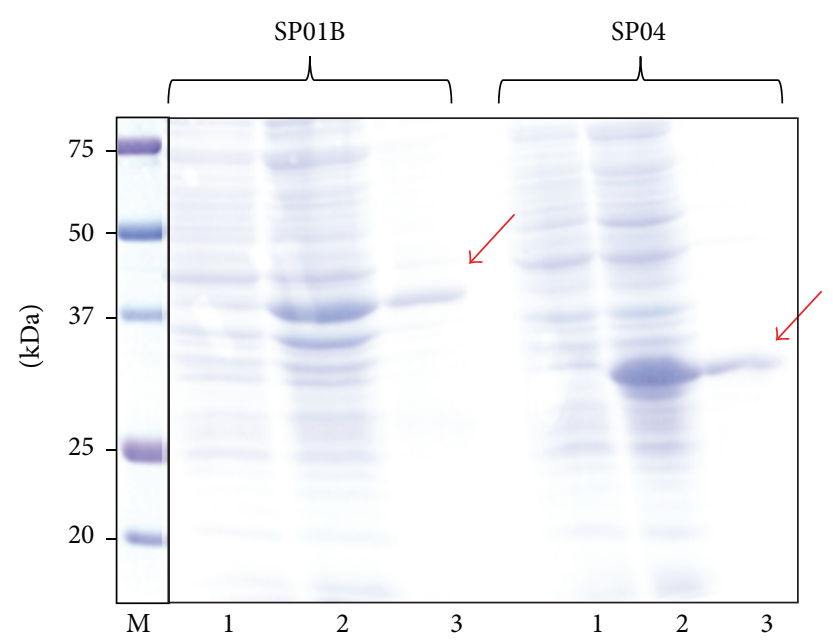

FIGURE 7: Coomassie blue-stained SDS-PAGE gel illustrating the expression and purification of the recombinant salivary proteins SP01B and SP04. Precision Plus Dual Xtra Standard (BioRad) was used as a marker (M). Lanes 1 and 2 show the bacterial extract before and after protein-expression induction by the addition of IPTG. Lane 3 shows the purified recombinant proteins.

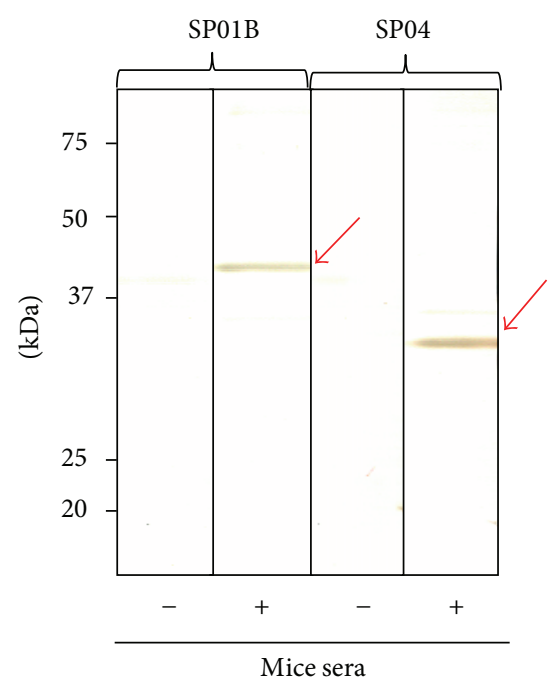

(a)

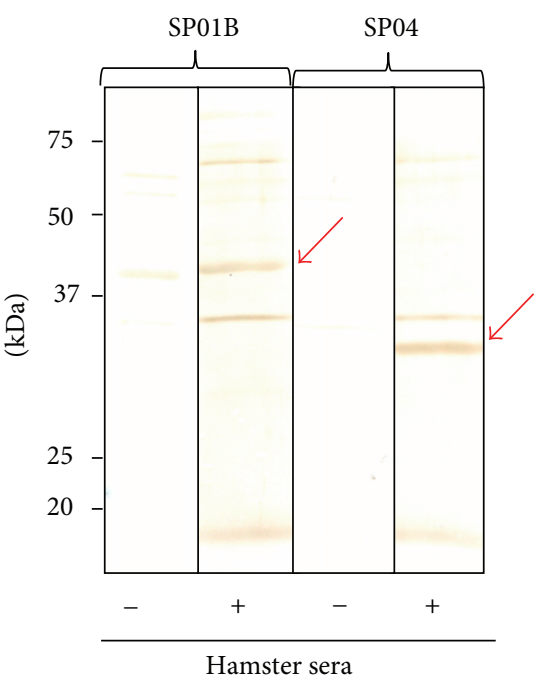

Hamster sera

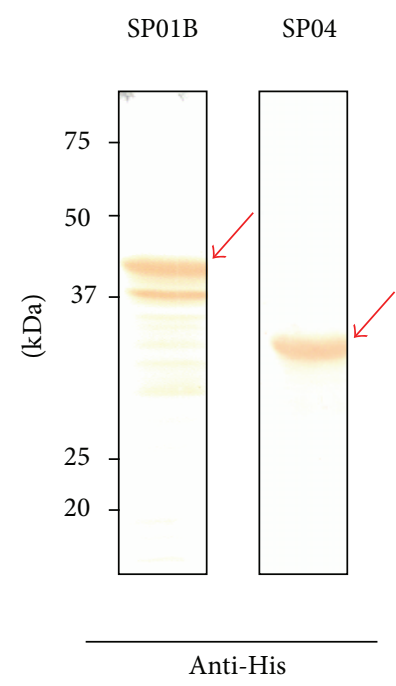

(b)

FIGURE 8: (a) Recognition of P. perniciosus recombinant salivary proteins SP01B and SP04 by Western blot using pooled sera of control and immunized mice and hamsters (marked as - and +, resp.). (b) Western blot with His antibodies confirmed the presence of the recombinant His-tagged proteins SP01B and SP04.

immunogenicity is due either to lineal epitopes or conformational ones after an appropriate refolding. Posttranslational modifications that may occur in nature might contribute to their immunogenicity but do not seem to be essential for the recognition of mice and hamster sera as the recombinant proteins lack them. Previously, we had found that SP01B was recognized by the sera of both hamsters and mice while SP04 was highlighted only by hamster sera [17]. However, in the current experiments, both recombinant proteins were recognized by hamster and mice sera. In the case of SP04, the discrepancy observed with previous results may be due to the differences in the schedule followed to immunize the mice (100 flies weekly over 10 weeks versus 13 times exposure to 150 flies). Moreover, the anti-saliva IgG levels from P. perniciosus mice immunized 13 times to 150 times were much higher than those sera from the other set of mice (data not shown).

\section{Conclusions}

Several polymorphisms were found between transcripts from the cDNA library of salivary glands of $P$. perniciosus and the cDNA library previously annotated [19], and further studies should be done in order to determine the biological meaning of all these polymorphisms. On the other hand, high level of conservancy was found between salivary transcripts of $P$. perniciosus from Spain and Italy thus, little antigenic variation is expected suggesting that recombinant salivary 
proteins could be used in different geographic areas where this sand fly species is present. Moreover, we successfully cloned, expressed, and purified SP01B and SP04 salivary proteins of $P$. perniciosus. Further characterization of these recombinant proteins will give additional information about their function, especially for SP04, as the functionality of the D7-related proteins has not been experimentally confirmed in sand flies. In addition, we preliminary tested the immunogenicity of these proteins with hyperimmune sera of mice and hamsters experimentally exposed to sand fly bites. Yet, these proteins should be further tested with sera of other reservoirs such as dogs, cats, hares, rabbits, and also with human sera in order to assess if they preserve immunogenicity for these species or not. In addition, further characterization of cellular immune responses of these recombinant proteins should be carried out to determine whether they could be selected as vaccine candidates against leishmaniasis.

Furthermore, to ensure that the best epidemiological markers are selected, it should be necessary to evaluate several recombinant proteins with sera of different hosts to select the most widely recognized proteins and test them alone or in combinations to cover a wide range of host immune responses. In this sense, additional works are in progress to obtain other recombinant proteins.

\section{Conflict of Interests}

The authors declare no conflict of interests.

\section{Acknowledgments}

This study was partially funded by the Spanish Ministry of Science \& Innovation (Project AGL2008-01592) and by EU grant FP7-2011-261504 EDENext and is catalogued by the EDENext Steering Committee as EDENext 082 (http://www.edenext.eu/). The contents of this paper are the sole responsibility of the authors and do not necessarily reflect the views of the European Commission. Inés Martín-Martín is a $\mathrm{Ph}$. D. recipient of a fellowship from the Spanish Ministry of Science \& Innovation (FPI-MICINN).

\section{References}

[1] WHO, Control of the Leishmaniases, Technical Report Series no. 949, World Health Organization, Geneva, Switzerland, 2010.

[2] J. M. C. Ribeiro and I. M. B. Francischetti, "Role of arthropod saliva in blood feeding: sialome and post-sialome perspectives," Annual Review of Entomology, vol. 48, pp. 73-88, 2003.

[3] S. Kamhawi, "The biological and immunomodulatory properties of sand fly saliva and its role in the establishment of Leishmania infections," Microbes and Infection, vol. 2, no. 14, pp. 1765-1773, 2000.

[4] A. Barral, E. Honda, A. Caldas et al., "Human immune response to sand fly salivary gland antigens: a useful epidemiological marker?" American Journal of Tropical Medicine and Hygiene, vol. 62, no. 6, pp. 740-745, 2000.

[5] R. Gomes and F. Oliveira, "The immune response to sand fly salivary proteins and its influence on Leishmania immunity," Frontiers in Immunology, vol. 3, article 110, 2012.
[6] I. Rohoušová and P. Volf, "Sand fly saliva: effects on host immune response and Leishmania transmission," Folia Parasitologica, vol. 53, no. 3, pp. 161-171, 2006.

[7] M. Vlková, I. Rohoušová, J. Drahota et al., "Canine antibody response to Phlebotomus perniciosus bites negatively correlates with the risk of Leishmania infantum transmission," PLoS Neglected Tropical Diseases, vol. 5, no. 10, article e1344, 2011.

[8] M. Vlková, I. Rohoušová, J. Hostomská et al., "Kinetics of antibody response in $\mathrm{BALB} / \mathrm{c}$ and $\mathrm{C} 57 \mathrm{BL} / 6$ mice bitten by Phlebotomus papatasi," PLoS Neglected Tropical Diseases, vol. 6, no. 7, article e1719, 2012.

[9] K. Gidwani, A. Picado, S. Rijal et al., "Serological markers of sand fly exposure to evaluate insecticidal nets against visceral leishmaniasis in India and Nepal: a cluster-randomized trial," PLoS Neglected Tropical Diseases, vol. 5, no. 9, article e1296, 2011.

[10] C. Teixeira, R. Gomes, N. Collin et al., "Discovery of markers of exposure specific to bites of Lutzomyia longipalpis, the vector of Leishmania infantum chagasiin Latin America," PLoS Neglected Tropical Diseases, vol. 4, no. 3, article e638, 2010.

[11] A. P. Souza, B. B. Andrade, D. Aquino et al., "Using recombinant proteins from Lutzomyia longipalpis saliva to estimate human vector exposure in visceral leishmaniasis endemic areas," PLoS Neglected Tropical Diseases, vol. 4, no. 3, article e649, 2010.

[12] M. F. Clements, K. Gidwani, R. Kumar et al., "Measurement of recent exposure to Phlebotomus argentipes, the vector of indian visceral leishmaniasis, by using human antibody responses to sand fly saliva," American Journal of Tropical Medicine and Hygiene, vol. 82, no. 5, pp. 801-807, 2010.

[13] J. G. Valenzuela, Y. Belkaid, E. Rowton, and J. M. C. Ribeiro, "The salivary apyrase of the blood-sucking sand fly Phlebotomus papatasi belongs to the novel Cimex family of apyrases," Journal of Experimental Biology, vol. 204, no. 2, pp. 229-237, 2001.

[14] S. Marzouki, M. Abdeladhim, C. B. Abdessalem et al., "Salivary antigen SP32 is the immunodominant target of the antibody response to Phlebotomus papatasi bites in humans," PLoS Neglected Tropical Diseases, vol. 6, no. 11, article e1911, 2012.

[15] R. Molina, M. I. Jiménez, I. Cruz et al., "The hare (Lepus granatensis) as potential sylvatic reservoir of Leishmania infantum in Spain," Veterinary Parasitology, vol. 190, no. 1-2, pp. 268271, 2012.

[16] M. Jiménez, E. González, A. Iriso et al., "Detection of Leishmania infantum and identification of blood meals in Phlebotomus perniciosus from a focus of human leishmaniasis in Madrid, Spain," Parasitology Research, vol. 112, no. 7, pp. 2453-2459, 2013.

[17] I. Martín-Martín, R. Molina, and M. Jiménez, "An insight into the Phlebotomus perniciosus saliva by a proteomic approach," Acta Tropica, vol. 123, no. 1, pp. 22-30, 2012.

[18] I. Martín-Martín, R. Molina, and M. Jiménez, "Identifying salivary antigens of Phlebotomus argentipes by a 2DE approach," Acta Tropica, vol. 126, no. 1, pp. 229-239, 2013.

[19] J. M. Anderson, F. Oliveira, S. Kamhawi et al., "Comparative salivary gland transcriptomics of sandfly vectors of visceral leishmaniasis," BMC Genomics, vol. 7, article 52, 2006.

[20] R. Molina, "Laboratory adaptation of an autochthonous colony of Phlebotomus perniciosus Newstead, 1911 (Diptera: Psychodidae)," Research and Reviews in Parasitology, vol. 51, no. 1-4, pp. 87-89, 1991.

[21] J. Sambrook and D. Russell, Molecular Cloning: A Laboratory Manual, Cold Spring Harbor Laboratory Press, New York, NY, USA, 2001. 
[22] R. Gupta, E. Jung, and S. Brunak, "Prediction of N-glycosylation sites in human proteins," in preparation, 2004, http://www.cbs .dtu.dk/services/NetNGlyc/.

[23] K. Julenius, A. Mølgaard, R. Gupta, and S. Brunak, "Prediction, conservation analysis, and structural characterization of mammalian mucin-type O-glycosylation sites," Glycobiology, vol. 15, no. 2, pp. 153-164, 2005.

[24] N. Blom, S. Gammeltoft, and S. Brunak, "Sequence and structure-based prediction of eukaryotic protein phosphorylation sites," Journal of Molecular Biology, vol. 294, no. 5, pp. 1351-1362, 1999.

[25] G. C. Lanzaro, A. H. C. S. Lopes, J. M. C. Ribeiro et al., "Variation in the salivary peptide, maxadilan, from species in the Lutzomyia longipalpis complex," Insect Molecular Biology, vol. 8, no. 2, pp. 267-275, 1999.

[26] R. S. Milleron, J.-P. Mutebi, S. Valle et al., "Antigenic diversity in maxadilan, a salivary protein from the sand fly vector of American visceral leishmaniasis," American Journal of Tropical Medicine and Hygiene, vol. 70, no. 3, pp. 286-293, 2004.

[27] H. Kato, J. M. Anderson, S. Kamhawi et al., "High degree of conservancy among secreted salivary gland proteins from two geographically distant Phlebotomus duboscqi sandflies populations (Mali and Kenya)," BMC Genomics, vol. 7, article 226, 2006.

[28] M. Abdeladhim, R. C. Jochim, M. Ben Ahmed et al., "Updating the salivary gland transcriptome of Phlebotomus papatasi (Tunisian Strain): the search for sand fly-secreted immunogenic proteins for humans," PLoS One, vol. 7, no. 11, Article ID e47347, 2012.

[29] S. S. Mahamdallie and P. D. Ready, "No recent adaptive selection on the apyrase of Mediterranean Phlebotomus: implications for using salivary peptides to vaccinate against canine leishmaniasis," Evolutionary Applications, vol. 5, no. 3, pp. 293-305, 2012.

[30] D.-E. A. Elnalem, C. Meneses, M. Slotman, and G. C. Lanzaro, "Genetic variation in the sand fly salivary protein, SP-15, a potential vaccine candidate against Leishmania major," Insect Molecular Biology, vol. 14, no. 2, pp. 145-150, 2005.

[31] A. Oleaga, A. Escudero-Población, E. Camafeita, and R. PérezSánchez, "A proteomic approach to the identification of salivary proteins from the argasid ticks Ornithodoros moubata and Ornithodoros erraticus," Insect Biochemistry and Molecular Biology, vol. 37, no. 11, pp. 1149-1159, 2007.

[32] I. M. B. Francischetti, V. M. Pham, B. J. Mans et al., "The transcriptome of the salivary glands of the female western black-legged tick Ixodes pacificus (Acari: Ixodidae)," Insect Biochemistry and Molecular Biology, vol. 35, no. 10, pp.1142-1161, 2005.

[33] L. Patthy, "Alternative splicing: evolution," eLS, 2008.

[34] C. Walsh, M. Gangloff, T. Monie et al., "Elucidation of the MD2/TLR4 interface required for signaling by lipid IVa," Journal of Immunology, vol. 181, no. 2, pp. 1245-1254, 2008.

[35] J. M. C. Ribeiro, B. J. Mans, and B. Arcà, "An insight into the sialome of blood-feeding Nematocera," Insect Biochemistry and Molecular Biology, vol. 40, no. 11, pp. 767-784, 2010.

[36] J. G. Valenzuela, R. Charlab, E. C. Gonzalez et al., "The D7 family of salivary proteins in blood sucking diptera," Insect Molecular Biology, vol. 11, no. 2, pp. 149-155, 2002.

[37] A. A. James, K. Blackmer, O. Marinotti, C. R. Ghosn, and J. V. Racioppi, "Isolation and characterization of the gene expressing the major salivary gland protein of the female mosquito, Aedes aegypti," Molecular and Biochemical Parasitology, vol. 44, no. 2, pp. 245-254, 1991.
[38] B. Arcà, F. Lombardo, A. Lanfrancotti et al., "A cluster of four D7-related genes is expressed in the salivary glands of the African malaria vector Anopheles gambiae," Insect Molecular Biology, vol. 11, no. 1, pp. 47-55, 2002.

[39] R. Charlab, J. G. Valenzuela, E. D. Rowton, and J. M. C. Ribeiro, "Toward an understanding of the biochemical and pharmacological complexity of the saliva of a hematophagous sand fly Lutzomyia longipalpis," Proceedings of the National Academy of Sciences of the United States of America, vol. 96, no. 26, pp. 15155-15160, 1999.

[40] E. Calvo, B. J. Mans, J. F. Andersen, and J. M. C. Ribeiro, "Function and evolution of a mosquito salivary protein family," Journal of Biological Chemistry, vol. 281, no. 4, pp. 1935-1942, 2006.

[41] I. Rohoušová, S. Subrahmanyam, V. Volfová et al., "Salivary gland transcriptomes and proteomes of Phlebotomus tobbi and Phlebotomus sergenti, vectors of leishmaniasis," PLoS Neglected Tropical Diseases, vol. 6, no. 5, article e1660, 2012.

[42] P. H. Alvarenga, I. M. B. Francischetti, E. Calvo, A. Sá-Nunes, J. M. C. Ribeiro, and J. F. Andersen, "The function and three-dimensional structure of a thromboxane A2/cysteinyl leukotriene-binding protein from the saliva of a mosquito vector of the malaria parasite," PLoS Biology, vol. 8, no. 11, Article ID e1000547, 2010.

[43] E. Calvo, B. J. Mans, J. M. C. Ribeiro, and J. F. Andersen, "Multifunctionality and mechanism of ligand binding in a mosquito antiinflammatory protein," Proceedings of the National Academy of Sciences of the United States of America, vol. 106, no. 10, pp. 3728-3733, 2009.

[44] J. Hostomská, V. Volfová, J. Mu et al., "Analysis of salivary transcripts and antigens of the sand fly Phlebotomus arabicus," BMC Genomics, vol. 10, article 282, 2009.

[45] J. G. Valenzuela, Y. Belkaid, M. K. Garfield et al., "Toward a defined anti-Leishmania vaccine targeting vector antigens: characterization of a protective salivary protein," Journal of Experimental Medicine, vol. 194, no. 3, pp. 331-342, 2001.

[46] P. K. Geyer, C. Spana, and V. G. Corces, "On the molecular mechanism of gypsy-induced mutations at the yellow locus of Drosophila melanogaster," EMBO Journal, vol. 5, no. 10, pp. 2657-2662, 1986.

[47] X. Xu, F. Oliveira, B. W. Chang et al., "Structure and function of a "yellow" protein from saliva of the sand fly Lutzomyia longipalpis that confers protective immunity against Leishmania major infection," Journal of Biological Chemistry, vol. 286, no. 37, pp. 32383-32393, 2011.

[48] F. Oliveira, S. Kamhawi, A. E. Seitz et al., "From transcriptome to immunome: identification of DTH inducing proteins from a Phlebotomus ariasi salivary gland cDNA library," Vaccine, vol. 24, no. 3, pp. 374-390, 2006.

[49] N. Collin, T. C. Assumpcao, D. M. Mizurini et al., "Lufaxin, a novel factor Xa inhibitor from the salivary gland of the sand fly Lutzomyia longipalpis blocks protease-activated receptor 2 activation and inhibits inflammation and thrombosis in vivo," Arteriosclerosis, Thrombosis, and Vascular Biology, vol. 32, no. 9, pp. 2185-2198, 2012.

[50] T. R. de Moura, F. Oliveira, M. W. Carneiro et al., "Functional transcriptomics of wild-caught Lutzomyia intermedia salivary glands: identification of a protective salivary protein against Leishmania braziliensis infection," PLoS Neglected Tropical Diseases, vol. 7, no. 5, article e2242, 2013. 
[51] H. Kato, R. C. Jochim, E. A. Gomez et al., "Analysis of salivary gland transcripts of the sand fly Lutzomyia ayacuchensis, a vector of Andean-type cutaneous leishmaniasis," Infection, Genetics and Evolution, vol. 13, pp. 56-66, 2012.

[52] J. Dai, J. Liu, Y. Deng, T. M. Smith, and M. Lu, "Structure and protein design of a human platelet function inhibitor," Cell, vol. 116, no. 5, pp. 649-659, 2004. 

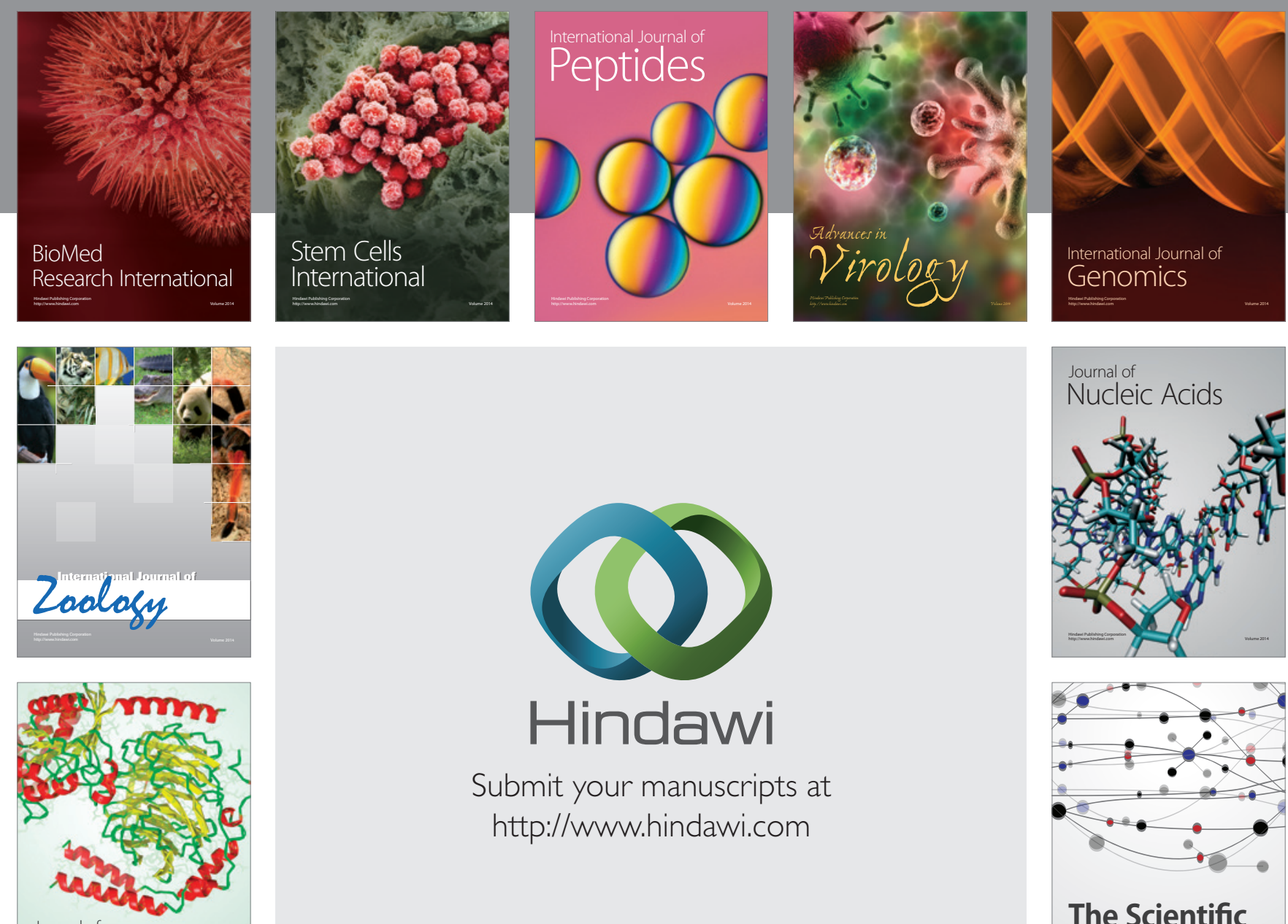

Submit your manuscripts at

http://www.hindawi.com

Journal of
Signal Transduction
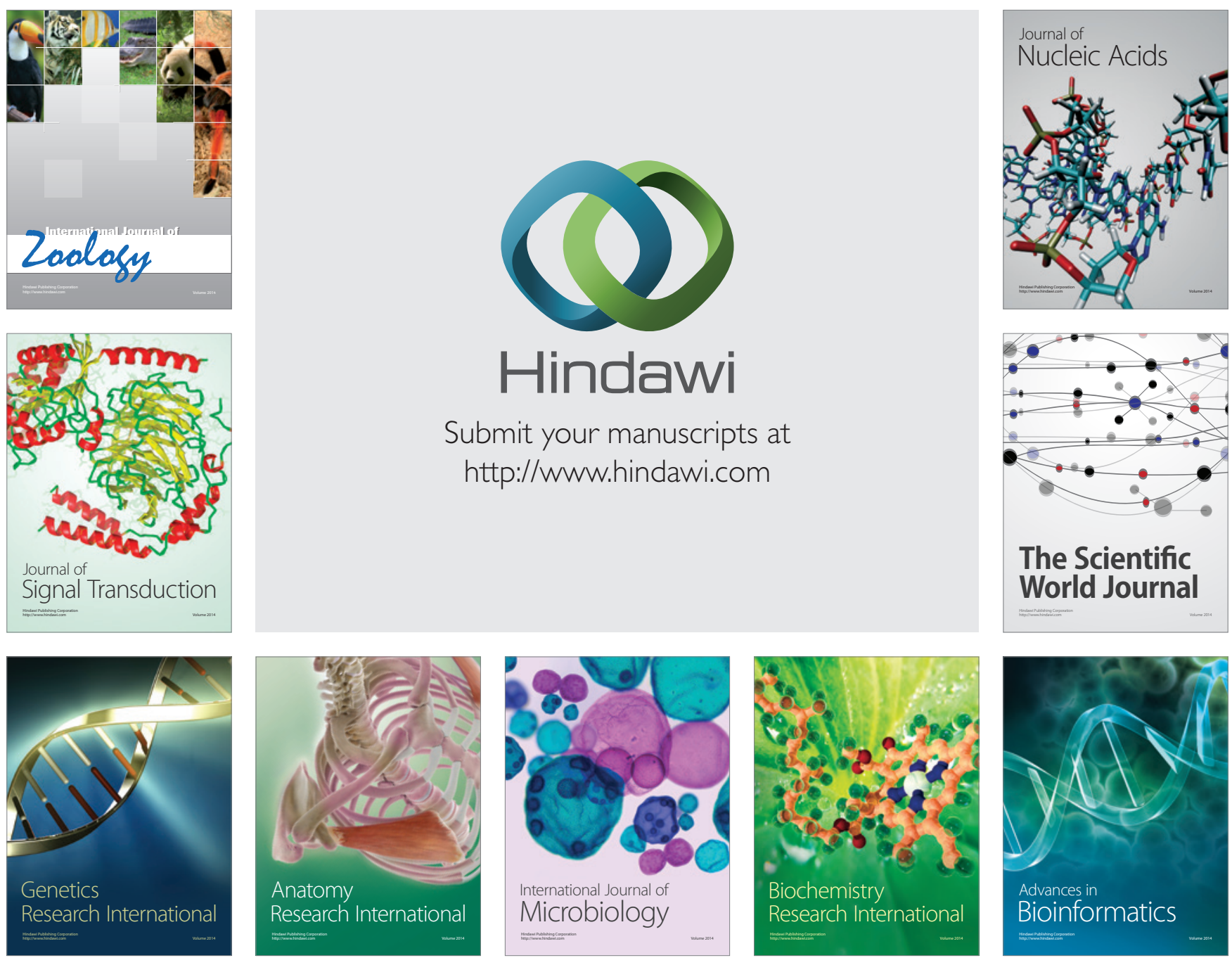

The Scientific World Journal
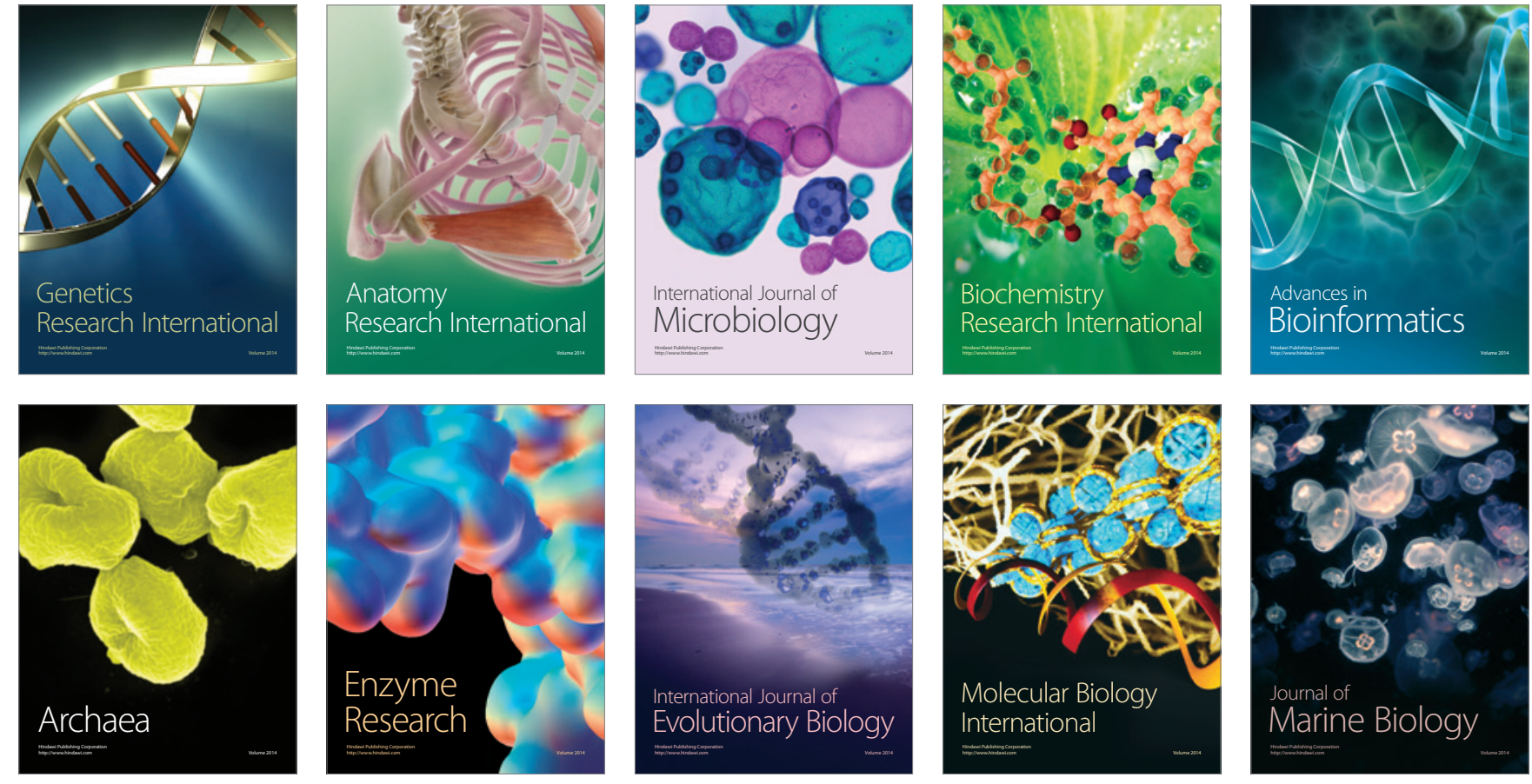